\title{
Special issue "Selected papers presented at the 2009 Spring meeting of the quantum optics and photonics section of the German Physical Society"
}

\author{
M. Fleischhauer
}

Published online: 30 January 2010

(c) Springer-Verlag 2010

The 2009 Spring Meeting of the AMOP Section of the German Physical Society, taking place at the University of Hamburg, was characterized by a lively exchange between the different areas of modern Atomic, Molecular, and Optical Physics.

The large number of joint sessions shared between the different Sections of the German Physical Society as well as seven interdisciplinary symposia demonstrated the dedication to research beyond the classical borders of disciplines.

The large number of oral and poster contributions within the Section Quantum Optics and Photonics which increased by more than 20 percent with respect to 2008 , reflects the increasing tendency to broaden the scientific goals and to explore new crossroads between different fields of physics. It was a pleasure to witness the high standards of the presentations, in particular the ones presented by enthusiastic diploma and doctoral students.

This special issue tries to convey a flavor of the wealth of presentations within the Quantum Optics and Photonics Section. The invited contributions, which all underwent the well-established refereeing process of Applied Physics B, were chosen on the basis of recommendations by the ses- sion chairs. It was the intention to cover a large variety of subjects.

Therefore the topics of this Special Issue range from fundamental problems to applications.

The articles address questions in quantum information from entanglement classification and

separability criteria to quantum cellular automata and atmospheric information channels.

Further subjects are properties of ultracold atoms and new detection methods for lattice gases.

New tools for the characterization of optical fibers and Lidar measurements are discussed as well as molecular excitations with few-cycle laser pulses. In the spirit of supporting young researchers, most of the corresponding authors of the articles in this Special Issue are at an early stage of their career.

Many thanks to the local organizers of the Hamburg meeting, in particular Markus Drescher, for realizing such an excellent conference. This Special Issue has been made possible by the professional support of the Editor-in-Chief Frank Träger and of Jutta Kaisig from the

University of Kassel, whom I also thank for her help.

M. Fleischhauer $(\bowtie)$

Fachbereich Physik und Landesforschungszentrum OPTIMAS,

Erwin-Schroedinger Str., 67663 Kaiserslautern, Germany

e-mail: mfleisch@physik.uni-kl.de 